\title{
EMPLOYEE STOCK OPTIONS INCENTIVE EFFECTS: A CPT-BASED MODEL
}

\author{
Hamza BAHAJI, DRM Finance,Université de Paris Dauphine, France \\ Email:hbahaji@yahoo.fr
}

\begin{abstract}
This paper examines the incentives from stock options for loss-averse employees subject to probability weighting. Employing the certainty equivalence principle, I built on insights from Cumulative Prospect Theory (CPT) to derive a continuous time model to value options from the perspective of a representative employee. Consistent with a growing body of empirical and experimental studies, the model predicts that the employee may overestimate the value of his options in-excess of their risk-neutral value. This is nevertheless in stark contrast with a common finding of standard models based on the Expected Utility Theory (EUT) framework that options value to a risk-averse undiversified employee is strictly lower than the value to risk-neutral outside investors. In particular, I proved that loss aversion and probability weighting have countervailing effects on the option subjective value. In addition, for typical setting of preferences parameters around the experimental estimates, and assuming the company is allowed to adjust existing compensation when making new stock option grants, the model predicts that incentives are maximized for strike prices set around the stock price at inception. This finding is consistent with companies' actual compensation practices that standard EUT-based models have difficulties accommodating their existence.
\end{abstract}

Keywords: Stock options, Cumulative Prospect Theory, Incentives, Subjective value.

JEL Classification: J33, J44, G13, G32, M12

\section{Introduction}

Instead of an increasing interest for restricted stock and performance unit plans, the 2006 Hewitt Associates Total Compensation Measurement survey has revealed that stock options are still the most prevalent long term incentive vehicle $^{1}$. The stated argument for the large use of executive stock options is that they align the interests of executives and shareholders since they provide incentives for the manager to act in order to increase the firm value. The use of stock options has even overtaken the traditional arena of executive population. Actually, firms' compensation practices show that stock options are issued to reward non-executive employees as well. In order to figure out the reason why stock options may be attractive to employees it is crucial to assess the utility they receive from them. Moreover, understanding how employee evaluates its stock options (i.e. their subjective value) allows assessing their incentive power and the implied employee behaviour in terms of risk taking.

Most of the theoretical literature on stock options relies on the Expected Utility theory (EUT henceforth) framework to derive models of option value from the employee perspective (Lambert et al, 1991; Hall and Merphy, 2000, 2002; Henderson, 2005). These models predict that the nontransferability of the options and the hedging restrictions faced by the employee make him value his options below their issuance cost born by the company (i.e. their risk neutral value). Moreover, standard normative models fail to predict stock options as part of the compensation contract. Several quantitative studies taking place in principal-agent framework showed that EUTbased models predict optimal compensation contracts which do not contain convex instruments like stock options (Holmstrom and Milgrom, 1987; Dittmann and Maug, 2007).

This paper analyzes the valuation of stock options and their incentives effect to an employee exhibiting preferences as described by Cumulative Prospect Theory (Tversky and Kahneman, 1992). It aims to propose an alternative theoretical framework for the analysis of pay-to-performance sensitivity of equity-based compensation that takes into account a number of prominent patterns of employee behaviour that standard EUT cannot explain. This work is motivated by recent empirical and theoretical researches on employee compensation incorporating CPT-based models (Dittmann et al., 2010; Spalt, 2008). These models have proved successful in explaining some observed compensation practices, and specifically the almost universal presence of stock options in the executive compensation contracts that the EUT models have difficulties accommodating their existence. Therefore, they have advanced CPT framework as a promising candidate for the analysis of equity-based compensation contracts.

\footnotetext{
${ }^{1} 80 \%$ of the responding participant companies to the survey have reported that stock option grants represent in 2006 , on average, about $54 \%$ of their global long-term incentives.
} 
I drew on this theoretical framework to derive a continuous time model of the stock option subjective value using the certainty equivalence principle. I then performed sensitivity analyses with respect to preferences-related parameters and found that loss aversion and probability weighting have countervailing effects. In particular, I proved that the option subjective value is increasing in probability weighting degree and decreasing in loss aversion. My analyses also show that, for a given level of option moneyness, the subjective value of the option may lies strictly above the Black and Scholes value (BS henceforth) when the effect of probability weighting tends to dominate that of loss-aversion. These results lead to the conclusion that the lottery-like nature of stock-options, combining large gains with small probabilities, may make them attractive to employees subject to probability weighting which is consistent with the proposition that employee option value estimate may exceed the BS value (Lambert and Larcker, 2001 ; Hodge et al., 2006 ; Sawers et al., 2006 ; Hallock and Olson, 2006 ; Devers et al., 2007).

Furthermore, this work elaborates on incentives from stock options and on some implications in terms of design aspects. Following previous researches, I defined incentives as the first order derivative of the subjective value with respect to the stock price. A numerical analysis of the incentive function shows that stock option incentive effects are increasing in employee's degree of probability weighting and may even lie above incentives for a risk-neutral individual. Moreover, I considered the incentive effects of setting the strike price of the option above or below the stock price at inception. In this analysis, I relied on Hall's and Murphy's (2002) methodology in solving for the exercise price that maximizes incentives holding constant the company cost of granting the options. I used this approach to explore the situation where the company is allowed to adjust existing compensation when making new stock option grants. For typical setting of preferences parameters around the experimental estimates from CPT (Tversky and Kahneman, 1992), the model predicts that incentives are maximized for strike prices set around the stock price at inception, which is consistent with companies' actual compensation practices. Additional analyses suggest also that loss-averse employees who are not subject to probability weighting, or even with very week degrees of probability weighting, receiving options at high exercise price would willingly accept a cut in compensation to receive instead deep discount options or restricted shares for those of them displaying more lossaversion. This result is broadly consistent with Hall and Murphy (2002) and Henderson (2005) findings for nondiversified risk-averse employees.

This article proceeds as follows. The first section describes the features of stock option value from the perspective of a representative employee with preferences as described by CPT. Throughout this paper, we will refer to this employee as a "CPT employee". This section provides also numerical analyses on the model sensitivity to preferences-related parameters. The next section introduces incentive effects of stock options for a CPT-employee and examines some design implications in terms of strike price setting. The risk taking incentives question is explored in the third and last section. Appendices provide proofs of the propositions in the first section.

\section{Stock option value from a CPT-employee perspective}

In this section, I develop a base-case model for analyzing the value of the stock-option contract from the perspective of a representative employee with CPT-based preferences (subjective value henceforth). Specifically, I assume that the employee is granted a European call option on the company's stock, denoted by $S$, with maturity date $t=T$ and strike price $K$. These are the traditional features of executive stock options as reported in Johnson and Tian (2000) and used by prior studies focused on stock option incentives (Lambert et al., 1991; Hall and Murphy, 2002; Henderson, 2005). Often in practice, stock options are Bermudan-style options. Thus, my model relies on a naïve setting in that it ignores complications related to early exercise or forfeitures.

\section{$2.1 \quad$ Theoretical framework}

\subsubsection{Stock-option contract}

The stock option contract is issued in $t=0$. The contract payoff at expiry, $t=T$, is $h_{T}=\left(S_{T}-K\right)^{+}$. I make the assumption that the employee is not allowed to short-sell the company stock and that he can earn the risk-free rate $r$ from investing in a riskless asset. Moreover, the price dynamic of the stock is given by a geometric Brownian motion represented by the following SDE:

$$
d S_{t}=(r-q) S_{t} d t+\sigma S_{t} d Z_{t}
$$


$Z_{t}$ is a standard Brownian motion with respect to the probability measure $I P . \sigma^{2}$ and $q$ are respectively the total variance of the stock price returns and the dividend yield.

\subsubsection{Risk preferences}

Following Tversky and Kahneman (1992), I consider that, to each gamble with a continuous random outcome $y \in I R$ which the probability density function is denoted by $f(y)$, the employee assigns the value:

$$
E_{\Omega}(y)=\int_{I R} v_{\theta}(y) d \Omega(f(y))
$$

Note that the expectation $E_{\Omega}($.$) is a function of two distinct functions. The first function, v_{\theta}($.$) , called the value$ function, is assumed of the form:

$$
v_{\theta}(y)=\left\{\begin{array}{c}
(y-\theta)^{\alpha} ; y \geq \theta \\
-\lambda(\theta-y)^{\alpha} ; y<\theta
\end{array}, \text { where } 0<\alpha \leq 1 \text { and } \lambda \geq 1\right.
$$

This formulation has some important features that distinguish it from the standard utility specification. First, the utility is defined over gains and losses assessed based on the reference point denoted by $\theta$. The second important feature is the shape of the value function. While it is convex over losses, it is concave over gains, which represents the observation from psychology that people are risk averse over gains and become risk-seeking over losses. Moreover, the value function has a kink at the origin introduced by the parameter $\lambda \geq 1$. This feature, known as loss-aversion, gives a higher sensitivity to losses compared to gains. Finally, outcomes are treated separately from other components of wealth, which reflects the well-documented phenomenon of narrow framing (Thaler, 1999).

The second function, $\Omega_{a, b}($.$) , is called the weighting function. It applies to cumulative probabilities, represented$ by the cumulative probability function $F($.$) , in order to transform them into decision weights according to:$

$$
\Omega_{a, b}(F(y))=\left\{\begin{array}{l}
\frac{-(1-F(y))^{a}}{\left(F(y)^{a}+(1-F(y))^{a}\right)^{\frac{1}{a}}} ; y \geq \theta \\
\frac{F(y)^{b}}{\left(F(y)^{b}+(1-F(y))^{b}\right)^{\frac{1}{b}}} ; y<\theta
\end{array} \quad \text { where } 0.28<a \leq 1 \text { and } 0.28<b \leq 1\right.
$$

This function stands for another piece of CPT, which is the nonlinear transformation of probabilities. Specifically, it captures experimental evidence on people overweighting small probabilities and being more sensitive to probability spreads at higher probability levels. The degree of probability weighting is controlled separately over gains and losses by the weighting parameters $a$ and $b$ respectively. The more these parameters approach the lower boundary at 0.28 the more the tails of probability distribution are overweighted. For instance, when $a=b=1$, probability weighting assumption is relaxed. For simplicity, these parameters are assumed to be equal (i.e. $a=b)$ and the weighting function will be denoted $\Omega_{a}($.$) in the rest of this paper. Finally, note that the lower boundary at 0.28$ is a technical condition to insure that $\frac{\partial \Omega_{a b}(p)}{\partial p}$ is positive over $] 0,1[$ as required by the following first order condition: $(a-1) p^{a}+(a(1-p)+p)(1-p)^{a} \geq 0$

\subsection{Stock option subjective value}

\subsubsection{The model}

In order to estimate the subjective value of the stock option contract described above, I use the certainty equivalence principle. In particular, this value is defined as the cash amount, $C_{\theta, a}$, that leaves the employee indifferent between 
this amount and the uncertain payoff of the contract, $h_{T}$, irrespective of the composition of the remainder of his private wealth. Formally, $C_{\theta, a}$ is the solution of the following equation:

$$
\begin{aligned}
v_{\theta}\left(C_{\theta, a} e^{r T}\right) & =E_{I P_{\Omega_{a}}}\left\{v_{\theta}\left(h_{T}\right)\right\} \\
& =\int_{I R} v_{\theta}\left(h_{T}\right) d \Omega_{a}\left(F\left(S_{T}\right)\right)
\end{aligned}
$$

The left-hand side of the equation above represents the benefit for the employee from receiving the cash amount $C_{\theta, a}$ instead of the stock option contract at the inception of the latter. This amount is assumed to be placed into the risk-free asset over the whole lifetime $T$ of the stock option contract. The other side of the equation gives the expected utility to the employee from receiving the risky payoff implied by the value function $v_{\theta}($.$) . Here, the$ expectation $E_{I P_{\Omega_{a}}}$ relies on the transformed probability measure $I P_{\Omega_{a}}$. Let $E_{\theta, a}$ represent the expectation in the righthand side of (5). It follows from (5) and (3) that:

$$
C_{\theta, a}=\left\{\begin{array}{l}
\left(\theta+\left(E_{\theta, a}\right)^{\frac{1}{\alpha}}\right) e^{-r T}, \text { if } E_{\theta, a} \geq 0 \\
\left(\theta-\left(-\frac{1}{\lambda} E_{\theta, a}\right)^{\frac{1}{\alpha}}\right) e^{-r T}, \text { otherwise }
\end{array}\right.
$$

Where $E_{\theta, a}$ should write:

$$
E_{\theta, a}=I_{\theta, a}^{1}+I_{\theta, a}^{2}+I_{\theta, a}^{3}
$$

With:

$$
\begin{gathered}
I_{\theta, a}^{1}=\int_{l_{1}}^{+\infty}(g(x)-K-\theta)^{\alpha} \Omega_{a}^{\prime}\left(\int_{-\infty}^{x} \varphi(u) d u\right) \varphi(x) d x \quad \text { (6.2); } I_{\theta, a}^{2}=\int_{l_{2}}^{l_{l}}-\lambda(K+\theta-g(x))^{\alpha} \Omega_{a}^{\prime}\left(\int_{-\infty}^{x} \varphi(u) d u\right) \varphi(x) d x \\
l_{1}=-\frac{\ln \left(\frac{S}{K+\theta}\right)+\left(r-q-\frac{\sigma^{2}}{2}\right) T}{\sigma \sqrt{T}} \quad(\mathbf{6 . 6}) ; l_{2}=-\frac{\ln \left(\frac{S}{K}\right)+\left(r-q-\frac{\sigma^{2}}{2}\right) T}{\sigma \sqrt{T}} \quad \text { (6.4) } g(x)=S e^{\left(r-q-\frac{\sigma^{2}}{2}\right) T+\sigma \sqrt{T} x}
\end{gathered}
$$

Here $\varphi($.$) is the Gaussian density function and \Omega_{a}^{\prime}(p)=\frac{\partial \Omega_{a}(p)}{\partial p}$ is the first-order partial derivative of $\Omega_{a}($.$) .$

\subsubsection{Reference point set up}

Although CPT specifies the shape of the value function around the reference point, it does not provide guidance on how people set their reference points. Neither does most of the psychological literature relying on the assumption according to which the reference point is the Status quo. Instead, this literature admits both the existence and the importance of non-status quo reference points since "there are situations in which gains and losses are coded relative to an expectation or aspiration level that differs from the status quo" (Kahneman and Tversky, 1979).

In principle, employee would update the reference point in a way that fits with his own expectations regarding the underlying share price at expiry. Intuitively, the employee could estimate the intrinsic value of the option based on his future share price forecasts as he can rely on the BS value of the option disclosed by the firm. Following Spalt (2008), I consider that the reference point parameter in the model, $\theta$, is the BS value ${ }^{2}$. Beyond the argument of empirical evidence on employee exercise behaviour depending on non-status quo reference points (Huddart and Lang, 1996; Heath et al, 1999; Bahaji, 2009), this assumption is supported by firms common practices in terms of

\footnotetext{
${ }^{2}$ To be more precise, the value used here is the expectation of the option payoff at expiring yielded by the BS model (i.e. the nondiscounted BS value). Consistent with this specification, the probability measure $I P$ used to derive the subjective value in (6) is the risk neutral probability measure. Moreover, ignoring the probability weighting feature (i.e. $a=1$ ), this setting allows the subjective value implied by the model to converge towards the risk neutral value (i.e. BS value) when the preferences of the employee tend to risk neutrality (i.e. $\alpha=\lambda=1$ ).
} 
stock option compensation. Most of stock-option designers use the BS model in order to estimate the value of stock options as constituents of the total compensation package. This value is usually announced to the employee at the inception of the options. Moreover, the BS model is recommended in the FASB and the IASC guidelines for determining the fair value of stock options (i.e. the amount an outside investor, with no hedge restrictions, would pay for the option) that needs to be disclosed in the financial statements. These statements, comprising the BS value of the stock options, are provided to shareholders as well as stakeholders including employees.

\subsubsection{The impacts of preferences-related parameters: a numerical analysis}

To provide a concrete outline on the profile of the subjective value yielded by the model relative to the risk neutral value profile, I performed a numerical analysis ${ }^{3}$ of the value of a 4 -year call option $(T=4)$ with a strike price $K=100$. For the remaining option-related parameters, the figures were computed assuming no dividend payments $(q=0 \%), \sigma=30 \%$ and $\mathrm{r}=3 \%$. Moreover, I set the curvature parameter of the value function $(\alpha)$ and the loss aversion coefficient $(\lambda)$ to respectively 0.88 and 2.25 based on experimental estimates from CPT (Tversky and Kahneman, 1992). Furthermore, in order to calibrate the probability weighting function, I used three different values of the parameter $a$ within the range of values estimated in the experimental literature ${ }^{4}$.

Figure 1 depicts the option value as a function of the stock price. The three blue curves represent the value profiles from the perspective of three CPT employees with the same value function and different degrees of probability weighting. At first sight, depending on the degree of probability weighting and the option moneyness $(S / K)$ the subjective value could lie either under or below the BS value. In contrast, standard EUT-based models predict that the option value from a risk-averse employee perspective is systematically lower than the risk-neutral value (Hall and Murphy, 2002; Henderson, 2005). These preliminary results are consistent thought with some empirical findings suggesting that frequently employees are inclined to overestimate the value of their stock-options compared with the BS value (Lambert and Larcker, 2001; Hodge et al., 2006; Sawers et al., 2006; Hallock and Olson, 2006; Devers et al., 2007).

The results presented in figure 1 show that the option subjective value is increasing in probability weighting degree (i.e. decreasing in parameter $a$ ). Actually, given the asymmetric profile of the option payoff, the expectation $E_{\theta, a}$ in the subjective value formula (6) is positively affected by the emphasis put on the tail of the payoff distribution which is governed by the parameter $a$ : the lower $a$ the more overweighed will be small probabilities and the more underweighted will be medium to large probabilities. This lottery-like nature of an option, combining large gains with small probabilities, may make it attractive to a CPT employee subject to probability weighting. This preliminary outcome leads to proposition 1 which stats that:

Proposition 1: the value of the stock option contract to a CPT-employee is increasing with respect to his degree of probability weighting (i.e. is decreasing with respect to a). (Proofs are provided in appendix A)

Moreover, the effect of probability weighting is expected to increase with the skewness of the distribution of the underlying stock price, which is captured by the volatility parameter $\sigma$ given the Log-normality assumed in the model. In order to show that, I performed a numerical analysis of the sensitivity of the subjective value to the probability weighting degree as a function of the volatility $\sigma$ and the parameter $a$. This sensitivity is defined as the partial derivative of subjective value with respect to $a$. The results are reported in figure 2 in the form of a graph. It shows that the sensitivity to parameter $a$ is negative and locally decreasing in volatility. That means that the more the share price is volatile the more the option will be attractive for a CPT employee subject to probability weighting. This supports Spalt's (2008) findings that the effect of probability weighting provides an economic rationale to riskier firms (i.e. more volatile firms) for granting more stock options to non-executive employees.

Furthermore, I investigate the effect of loss aversion on the subjective value. The variable of interest here is $\lambda$. In an analogical sense with the EUT framework, the option value from the perspective of a loss-averse employee is expected to decrease with his degree of loss-aversion. To verify this I computed numerically the first order derivative with respect to $\lambda$ crossed over various levels of $\lambda$ and moneyness, ranging from 0.05 to 1 and from $5 \%$ to

\footnotetext{
${ }^{3}$ The integrals in (6.2), (6.3) and (6.4) were computed numerically.

${ }^{4}$ Tversky and Kahneman (1992) got 0.65 on average ( $a=0.61$ for gains and $b=0.69$ for losses). These results are corroborated by Abdellaoui's (2000) findings ( $a=0.60$ for gains and $b=0.70$ for losses, hence an average of 0.65$)$. In addition, Camerer and Ho (1994) obtained $a=0.56$ for gains whereas Gonzales and Wu (1996) and Bleichrodt and Pinto (2000) found $a=0.71$ and $a=0.67$ respectively.
} 
$200 \%$ respectively. The outcome is reported in figure 3 . It shows that the sensitivity to loss aversion is negative and locally decreasing in moneyness. That means that the more the employee is loss averse, the less would the option be worth to him. This conclusion is taken up in proposition 2 hereafter:

Proposition 2: the value of the stock option contract to a CPT-employee is decreasing with respect to his degree of loss-aversion (i.e. a decreasing function of $\lambda$ ). (Proofs are provided in appendix B)

I performed a similar analysis in order to get a view on the effect of the curvature parameter $\alpha$. In the same way, I assessed locally the first order derivative with respect to $\alpha$ within a range of values from 0.05 to 1 and using share prices ranging from 10 to 200 . While not formally reported in this paper, the results show that this derivative is locally increasing with the option moneyness for values of $\alpha$ above say 0.75 . It also shows broadly that the subjective value is an increasing monotone function of $\alpha$ over a range of values around the experimental estimate of 0.88 (from 0.7 to 1 ) irrespective of the option moneyness.

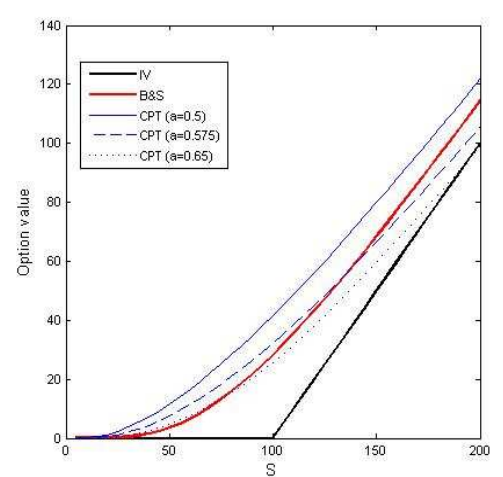

Figure-1: Option value against the stock price. This figure is a plot of the subjective value computed under different probability weighting parameters (the blue curves). It illustrates the profile of the subjective value compared to that of the risk neutral value (red curve). The Parameters used are: $\mathrm{T}=4 ; \quad \mathrm{K}=100 ; \quad \sigma=30 \% ; \quad \mathrm{r}=3 \% ; \quad \mathrm{q}=0 \%$;

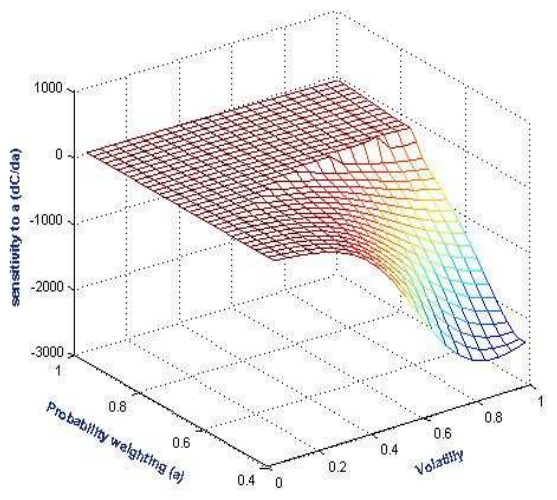

Figure-2: Sensitivity to probability weighting. This figure is a plot of the partial derivative of the subjective value with respect to "a" against both "a" and the stock price volatility " $\sigma$ ". It exhibits the local effect of probability weighting given the payoff distribution skewness captured by " $\sigma$ ". The derivative was computed numerically based on the following parameters: $\mathrm{T}=4 ; \mathrm{K}=\mathrm{S}=100$; $\mathrm{r}=3 \% ; \mathrm{q}=0 \% ; \lambda=2.25 ; \alpha=0.88$.

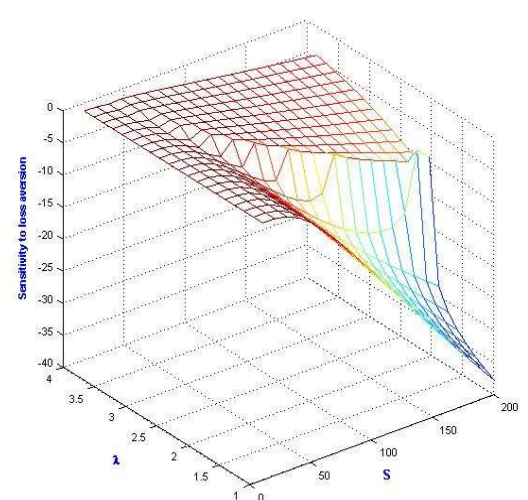

Figure-3: Sensitivity to loss aversion. This figure is a plot of the partial derivative of the subjective value with respect to " $\lambda$ " against both " $\lambda$ " and the stock price "S". It exhibits the local effect of loss aversion given the option moneyness. The derivative was computed numerically based on the following parameters: $\mathrm{T}=4 ; \quad \sigma=30 \%$; $\mathrm{K}=100 ; \mathrm{r}=3 \% ; \mathrm{q}=0 \% ; \mathrm{a}=0.65 ; \alpha=0.88$.

\section{$3 \quad$ Incentives from stock-options}

Stock-options are incentive tools used within a principal-agent relationship to align the interests of the agent (employee) on those of the principal (shareholders). The shareholders grant stock options in order to provide the employee with incentives to make efforts that enhance the value of the firm, and thus their own wealth. Indeed, assuming that employees are aware of how their actions affect the share price, option holdings will prompt them to make efforts that increase share price. Therefore, the incentive from a single option grant will depend on the degree of the sensitivity of the subjective value to the stock price.

\subsection{The incentive measure}

Following Jensen and Murphy (1990), Hall and Murphy (2000, 2002) and others, I defined the incentive effect as the first order derivative of the subjective value with respect to share price which defines how the value from the employee perspective changes with an incremental change in the stock price. A preliminary numerical analysis 
relying on the setting reported in $\$ 2.2 .3$ shows that incentives are greatest for in-the-money ${ }^{5}$ options and increasing with the degree of probability weighting. Another result from the analysis is that, for sufficiently high level of probability weighting, the option can give much more incentive to increase stock price than is reflected by the BS delta. This is consistent with my previous finding that the subjective value can overstate the BS value. In addition, consistently with the EUT-based models, the analysis results suggest that when the employee process probabilities in a linear way $(a=1)$ - which means that the probability weighting assumption is relaxed and only loss-aversion matters - the incentives lie strictly under the BS delta whatever the level of the option moneyness. That is to say that the options are less attractive for both risk-averse employee and loss-averse employee who are not subject to probability weighting. Furthermore, with $a$ hold constant at 0.65 , I find that incentives are decreasing in lossaversion. Conversely, with loss-aversion parameter $\lambda$ set to 1 , which means that the loss-aversion effect is neutralized, incentives for a representative loss-neutral employee, with a degree of probability weighting equal to experimental estimate of 0.65 , overstate the BS delta.

\subsection{Implications for stock option design: optimal strike price}

Setting the strike price of standard stock options boils down to defining the threshold against which the performance is assessed and, consequently, to determining the likelihood of a final payout. As stated in the previous section, incentives increases in the option moneyness and, equivalently, decreases with strike price. In parallel, from the shareholders perspective, granting in-the-money options is much more costly than granting out-of-the money or atthe-money options (recall figure 1). This leads the firm to a trade-off to make when setting the exercise price of the options in the sense that, holding his cost unchanged, she could either grant fewer options at a low strike price or increase the grant size at higher exercise price.

I relied on the methodology from Hall and Murphy (2002) in figuring out the optimal exercise price satisfying the double purpose of maximizing incentives and holding constant the firm's cost of granting options. I considered the situation where the employee and the firm are allowed to bargain efficiently over the terms of the compensation. Thus, the firm is assumed to fund additional options by an adjustment to other compensation components that leaves the employee indifferent between his initial package and the new package including the additional grant.

Let's considerer then that the company is allowed to make an efficient adjustment to existing compensation components (cash for example) to grant additional options to the employee. The impact of this adjustment should be neutral with regard to the total compensation cost for the company. Moreover, assuming this adjustment involves cash compensation, it must be attractive to the employee so that he'd be willing to give up some cash compensation against extra options grant. Therefore, it must leave the employee at his initial total subjective value of the compensation package. It follows that the strike price that maximizes total incentives for a given company cost is the solution of this following optimization problem:

$$
\max _{K} \frac{\partial n C_{\theta, a}(K)}{\partial S} \text { subject to } n \pi(K)-n C_{\theta, a}(K)=c \text { and } n>0
$$

Where $\frac{\partial n C_{\theta, a}(K)}{\partial S}$ denotes the incentives from receiving $n$ options with a strike price $K, \pi(K)$ is the per-unit cost or the BS value of one option and $c$ is a fixed constant. The constraint in (7) is the aggregation of the company cost constraint and the employee value constraint used in Henderson (2005). This optimization problem was solved numerically by varying the parameter $K$. First, the BS and the subjective values are computed for a given $K$ which enables to determine the grant size $n$ in accordance with the constraint in (7) for $K \neq S$. Then, the cost function is assessed based on $n$ and $K$. This procedure is reiterated recursively until the optimal value of $K$ is found. In this analysis, $c$ was chosen such that for retained parameters, the number of granted at-the-money options $n$ is around 1000 , hence a total cost of $€ 28333$.

The left-hand side sub-figure in figure- 4 exhibits total incentives for different levels of $K$ and probability weighting parameter $a$, with risk aversion held constant at $\lambda=2.25$. For each combination of $K$ and $a$ the constraint in (7) is solved for $n$, which allows to determine total incentives. The plots indicate that when the employee is deeply

\footnotetext{
${ }^{5}$ The terminology "at-the-money" is referring to stock-options with an exercise price equal to the stock price at inception. The expressions "Outof- the-money" and "In-the-money" are also used throughout the paper to refer to options with strike price respectively below and above the grant date stock price.
} 
subject to probability weighting ( $a \leq 0.475)$, total incentives are strictly decreasing throughout the depicted range of strike prices (see the curves in blue). In this case, similar to EUT-based studies' findings (Hall and Murphy, 2002; Henderson, 2005), incentives are maximized through restricted stocks grant rather than stock options. The intuitive reason behind that is that, given that the employee systematically values the options in excess of their BS value, efficient trade-off over compensation allocation is made via the grant of equity-based instruments that employee values at their actual cost, restricted stocks for instance. However, for lower degrees of probability weighting (typically $0.475<a \leq 0.85$ ) the optimal strike price is not nil and tends to increase with $a$. Specifically, the optimization problem is defined on the right (see the red solid curve) or on the left (see the red dashed curve) of the optimal strike $K^{*}$ and the cost function admits an infinite branch in $K^{*}$ (i.e. total incentives are infinite at that point). The technical reason behind that is that the subjective value is equal to the BS value at the optimal strike, which yields according to the constraint in (7) an infinite grant size $\left(\lim _{K \rightarrow K^{*}} n(K)=+\infty\right)$. For instance, $K^{*}$ ranges from $60 \%$ to $195 \%$ when $a$ takes values within a range around the experimental estimates $(0.55<a \leq 0.70)^{6}$. This suggests that, depending on the extent to which employee weights probabilities, either grants of discount options or premium options may result in a Pareto optimum to the contract. As a specific example, for an employee with $a=0.616$, the optimal strike price $K^{*}$ is around $100 \%$, which means that, for the prevailing setting, the model predicts at-the-money options as optimal in an efficient contracting framework since the company's cost of granting these options is equal to their value to the employee. In this case, granting options with strike price around the stock price at inception, which is consistent with observed practices, results in a perfect reconciliation of the company and the employee respective views. Note that for a similar setting in the previous situation where the options are given as an add-on to existing compensation, the model indicates that incentives from stock options could be infinitely increased by granting increasingly more options at greater and greater strike price.
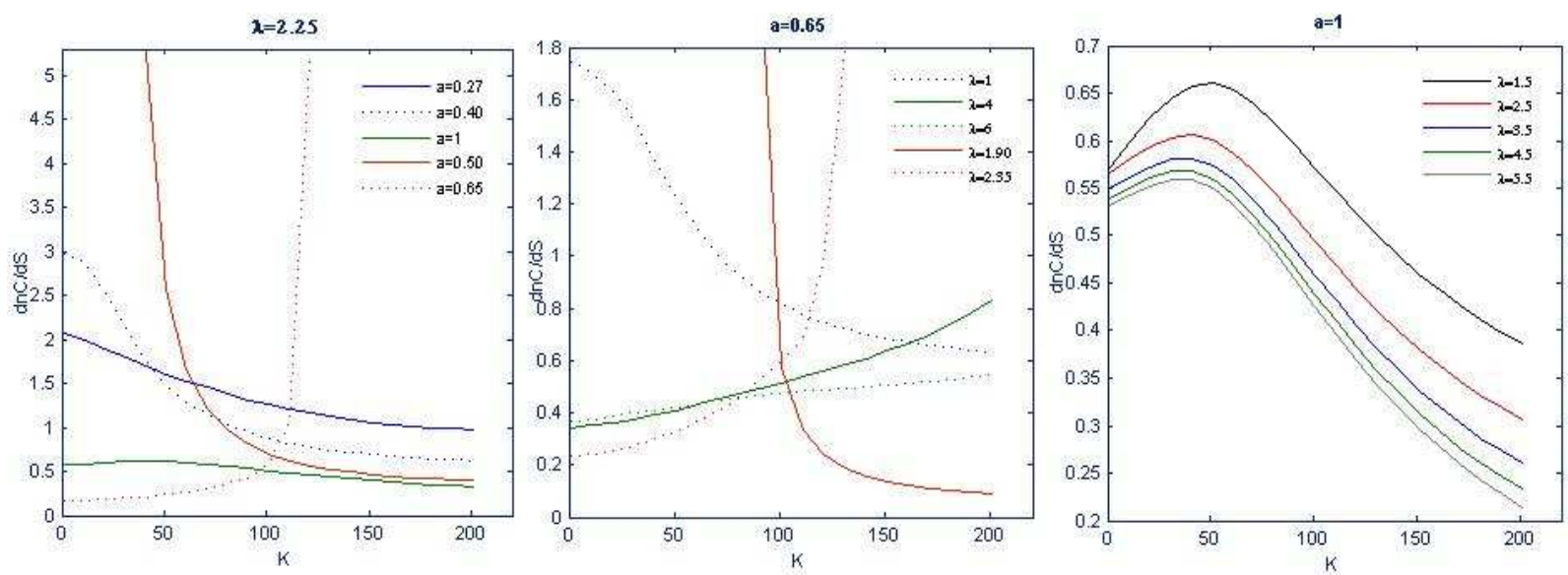

Figure-4. Incentives from options grant with total cost of $€ 28333$ assuming cash compensation is adjusted. This figure is plots of incentives (in $\mathrm{K} €$ ) from options grant for a given strike price holding the total compensation value constant from both company and employee perspectives. Total incentives are defined as the partial derivative of the total subjective value of the grant with respect to stock price " $S$ ". The sub-figure on the left-hand side depicts total incentives as a function of the strike price for varying levels of probability weighting parameter. That in the middle is a plot of total incentives against strike price for varying levels of loss-aversion. The sub-figure on the righthand side exhibits the sole effect of loss-aversion (with no probability weighting) on the shape of total incentives as a function of strike price. The derivatives were computed numerically based on the following parameters: $\mathrm{T}=4 ; \sigma=30 \% ; \mathrm{S}=100 ; \mathrm{r}=3 \% ; \mathrm{q}=0 \% ; \alpha=0.88$.

Furthermore, for weak degrees of probability weighting $(0.85<a)$, the subjective value of the option lies strictly below the BS value because the effect of loss-aversion tends to dominate that of probability weighting. Thus the total incentives function takes a non-monotone concave shape and shows finite extremas ranging from $35 \%$ to $81 \%$. In particular, when probability weighting assumption is relaxed $(a=1)$, the function flattens around the maximum and approaches a monotone decreasing shape with increasing loss-aversion (see sub-figure on the right-hand side). This result suggests that loss-averse employees who are not subject to probability weighting - even with very low degree of probability weighting - receiving options at high exercise price would willingly accept a cut in cash compensation to receive instead deep discount options or restricted shares for those of them displaying more loss-

\footnotetext{
${ }^{6}$ Optimal strikes are expected to be lower for higher stock price volatility since probability weighting effect tends to increase with volatility (recall figure 2).
} 
aversion. Again, this is broadly consistent with Hall's and Murphy's (2002) and Henderson's (2005) findings for non-diversified risk-averse employees.

Last but not least, the sub-figure in the middle of the main figure-4 is plots of total incentives against the strike price; each of them corresponds to a given level of loss-aversion. The purpose of this analysis consists in showing the effect of loss-aversion on $K^{*}$ by holding $a$ constant at 0.65 and varying $\lambda$. It mainly shows that loss-aversion has an effect opposite to that of probability weighting: when probability weighting effect is dominant ${ }^{7}$ (i.e. the employee may potentially put overstated value in the option, specifically for high strikes) optimal strike increases and total incentives decreased with loss-aversion. Conversely, when loss-aversion effect is dominant, as stated before, the model yields predictions comparable to that of the EUT-based models.

\section{Conclusion}

This paper proposes an alternative theoretical model of stock option subjective value to analyze their incentive effects for employees. The model predictions ascertain the ability of CPT to explain some prominent incentive patterns that EUT models have difficulties to capture. It mainly provides arguments on the well documented tendency of employees to frequently value - under some circumstances - their options in excess of their cost to the company. Specifically, these results highlight the economic rational for firms, in particular those with higher risk, to widely use stock options in non-executive employee compensation (Spalt, 2008).

Loss aversion and probability weighting are the key features driving the subjective value in the CPT model. These parameters have countervailing effects on the modeled subjective value. Depending on which of them is dominant in the preferences calibration, the model yields different predictions regarding incentives. Specifically, consistent with some behavioural patterns observed in many surveys and experimental studies on equity-based compensation, the model predicts that, when the probability weighting feature prevails, the subjective value may overstate the risk-neutral value of the option. In this case, assuming the company and the employee bargain efficiently over the compensation components, incentives are maximized for strike prices set around the stock price at inception for a representative employee with preferences calibration meeting the experimental estimates from CPT (Tversky and Kahneman, 1992). Obviously, this finding is consistent with companies' actual compensation practices. Moreover, executives with such preferences profile may be prompted to act in order to increase share price volatility. However, when the emphasis is put on the loss-aversion feature, by relaxing the probability weighting assumption, the model yields results comparable to those of EUT-based models. In particular, the model predicts that loss-averse employees who are not subject to probability weighting, or even with very low degrees of probability weighting, receiving options at high exercise price would willingly accept a cut in compensation to receive instead deep discount options or restricted shares for those of them displaying more loss-aversion.

Despite their practical interest, the conclusions from the results of this research should not be drawn without underlining some of its limitations. The first one concerns the specification of the CPT model. Actually, although the reference point specification in the model is consistent with both empirical evidence on people setting reference points in a dynamic fashion and firms' widespread use of the BS value as a standard for Financial and Human Resources disclosers, empirical and experimental literature is still silent on how people set reference points when assessing complex gambles like stock option payoffs. In addition, to keep the model tractable, only European-style options were studied in this paper. The model is still however easily extendable to Bermudian-style options using numerical schemes such like lattice approaches. The other limitation of this study is related to the large heterogeneity in probability weighting that may exist across individuals (Wu and Gonzalez, 1996). The Tversky's and Kahneman's (1992) weighting function used in the model provides only a fit to the median profile.

Finally, this work highlights - as did some previous eminent researches in this field (Dittmann et al., 2010; Splat, 2008) - a number of future promising research directions in equity-based compensation incorporating CPT framework. For instance, exploring the ability of CTP to explain the growing use of performance shares plans instead of stock options in employee compensation would be of great interest for future research. Furthermore, given that several empirical studies has documented that employee stock option exercise behaviour is also driven by

\footnotetext{
${ }^{7}$ See the limit case of $\lambda=1$ where risk-aversion is ignored (the blue dashed curve).
} 
behavioural factors, a promising research direction incorporating CPT is studying its ability to predict exercise patterns.

\section{$5 \quad$ Appendices}

\subsection{Appendix A: proofs of proposition 1}

This appendix provides heuristic proofs of proposition 1 . To prove that the subjective value $C_{\theta, a}$ is decreasing in $a \in[0.28,1]$, it is sufficient to show that the expectation $E_{\theta, a}$ is a decreasing function of $a$. Let's first rewrite the expectation in (6) as follows:

$E_{I P_{\Omega_{u}}}\left\{v_{\theta}\left(h_{T}\right)\right\}=E_{I P_{\Omega_{u}}}\left\{v_{\theta} \circ h_{T} \circ g(x)\right\}=\int_{I R} w_{\theta}(x) \Omega_{a}^{\prime}\left(\int_{-\infty}^{x} \varphi(u) d u\right) \varphi(x) d x$

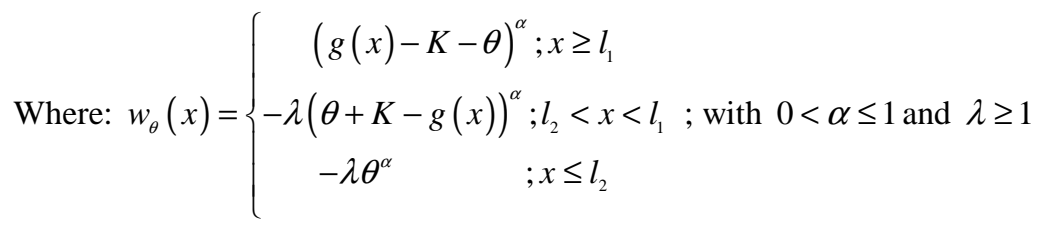

Since $w_{\theta}(x)$ is derivable over $I R \backslash\left\{l_{1}\right\}$, an expansion in Taylor series at the first order gives:

$w_{\theta}(x) \approx w_{\theta}(y)+(x-y) w_{\theta}^{\prime}(y)$; with $y \in I R \backslash\left\{l_{1}\right\}$

Introducing the expectation operator with respect to the transformed probability measure, we have:

$$
E_{I P_{\Omega_{u}}}\left(w_{\theta}(x)\right) \approx w_{\theta}(y)+\left(E_{I P_{\Omega_{u}}}(x)-y\right) w_{\theta}^{\prime}(y)=w_{\theta}(y)+\left(E_{I P}\left(x \Omega_{a}^{\prime}\left(\int_{-\infty}^{x} \varphi(u) d u\right)\right)-y\right) w_{\theta}^{\prime}(y)
$$

Since $w_{\theta}(x)$ is monotone increasing in $x$, we have: $w_{\theta}^{\prime}(y) \geq 0$. Then it follows from (A.2) that proving that $E_{\theta, a}$ is locally decreasing with respect to $a$ in $y$ boils down to showing that:

$$
\frac{\partial E_{I P}\left(x \Omega_{a}^{\prime}\left(\int_{-\infty}^{x} \varphi(u) d u\right)\right)}{\partial a} \leq 0
$$

Noting that $\Omega_{a}^{\prime}\left(\int_{-\infty}^{x} \varphi(u) d u\right)$ is positive and convex with respect to $\left.x \in\right]-\infty, l_{1}[\cup] l_{1},+\infty[$, we have according to Jensen inequality: $E_{I P}\left(x \Omega_{a}^{\prime}\left(\int_{-\infty}^{x} \varphi(u) d u\right)\right) \geq E_{I P}(x) \Omega_{a}^{\prime}\left(\int_{-\infty}^{E_{I P}(x)} \varphi(u) d u\right)$

Since $x$ follows a standard normal distribution, which is symmetric in $E_{I P}(x)=0$, we get from the inequality above, by substituting $E_{I P}(x)$ in the right hand side:

$E_{I P}\left(x \Omega_{a}^{\prime}\left(\int_{-\infty}^{x} \varphi(u) d u\right)\right) \geq 0$

Note that the result in (A.4) above, showing that the expectation under the transformed probabilities overstates that under the original probabilities, is a consequence of the probability weighting function feature stated in $\S 1.1 .2$, which consists in infinitely-overweighting infinitesimal probabilities and infinitely-underweighting near-one probabilities.

Furthermore, noting that the convexity of $\Omega_{a}^{\prime}\left(\int_{-\infty}^{x} \varphi(u) d u\right)$ with respect to $\left.x \in\right]-\infty, l_{1}[\cup] l_{1},+\infty[$ is decreasing in $a$, and using the result in (A.4) we have for $0.28<a<b \leq 1$ : 
$E_{I P}\left(x \Omega_{a}^{\prime}\left(\int_{-\infty}^{x} \varphi(u) d u\right)\right)-E_{I P}(x) \Omega_{a}^{\prime}\left(\int_{-\infty}^{E_{\mu}(x)} \varphi(u) d u\right) \geq E_{I P}\left(x \Omega_{b}^{\prime}\left(\int_{-\infty}^{x} \varphi(u) d u\right)\right)-E_{I P}(x) \Omega_{b}^{\prime}\left(\int_{-\infty}^{E_{t \prime}(x)} \varphi(u) d u\right)$

Or equivalently:

$E_{I P}\left(x \Omega_{a}^{\prime}\left(\int_{-\infty}^{x} \varphi(u) d u\right)\right) \geq E_{I P}\left(x \Omega_{b}^{\prime}\left(\int_{-\infty}^{x} \varphi(u) d u\right)\right)$

This proves that the condition in (A.3) holds, which means that $C_{\theta, a}$ is a decreasing function of $a$. Q.E.D.

\subsection{Appendix B: proofs of proposition 2}

Taking the derivative of the subjective value function in (6) with respect to $\lambda$ yields:

$\frac{\partial C_{\theta, a}}{\partial \lambda}=\left\{\begin{array}{l}\frac{1}{\alpha} \frac{\partial E_{\theta, a}}{\partial \lambda}\left(E_{\theta, a}\right)^{\frac{1}{\alpha}-1} e^{-r T}, \text { if } E_{\theta, a} \geq 0 \\ -\frac{1}{\alpha \lambda}\left(-\frac{E_{\theta, a}}{\lambda}\right)^{\frac{1}{\alpha}-1}\left(\frac{E_{\theta, a}}{\lambda}-\frac{\partial E_{\theta, a}}{\partial \lambda}\right) e^{-r T} \text {, otherwise }\end{array}\right.$

Where: $\frac{\partial E_{\theta, a}}{\partial \lambda}=\frac{\partial I_{\theta, a}^{1}}{\partial \lambda}+\frac{\partial I_{\theta, a}^{2}}{\partial \lambda}+\frac{\partial I_{\theta, a}^{3}}{\partial \lambda}$

Using the formulas in equations from (6.2) to (6.4), we can rewrite the derivatives in (B.2) as follows:

$\frac{\partial I_{\theta, a}^{1}}{\partial \lambda}=0$

$\frac{\partial I_{\theta, a}^{2}}{\partial \lambda}=-\int_{l_{2}}^{L_{1}}(K+\theta-g(x))^{\alpha} \Omega_{a}^{\prime}\left(\int_{-\infty}^{x} \varphi(u) d u\right) \varphi(x) d x$

$\frac{\partial I_{\theta, a}^{3}}{\partial \lambda}=-\theta^{\alpha} \Omega_{a}\left(\int_{-\infty}^{b} \varphi(x) d x\right)$

Then substituting these results in place of the derivatives in (B.2) we obtain:

$\frac{\partial E_{\theta, a}}{\partial \lambda}=-\int_{l_{2}}^{l_{l}}(K+\theta-g(x))^{\alpha} \Omega_{a}^{\prime}\left(\int_{-\infty}^{x} \varphi(u) d u\right) \varphi(x) d x-\theta^{\alpha} \Omega_{a}^{\prime}\left(\int_{-\infty}^{l_{2}} \varphi(x) d x\right)$

Given that $\frac{\partial E_{\theta, a}}{\partial \lambda} \leq 0$, we conclude that $\frac{\partial C_{\theta, a}}{\partial \lambda}$ is nonpositive. This implies that $C_{\theta, a}$ is a decreasing function of $\lambda$. Q.E.D.

\section{References}

Abdellaoui, M. (2000), "Parameter-Free Elicitation of Utility and Probability Weighting Functions", Management Science, Vol. 46 No. 11, pp. 1497-1512.

Bahaji, H. (2009), "Le comportement d'exercice des porteurs de stock-options: une étude empirique sur le marché Américain”, Working Paper, DRM Finance- Université de Paris Dauphine, Paris, June 2009.

Bleichrodt, H. and Pinto, J.L. (2000), "A Parameter-Free Elicitation of the Probability Weighting Function in Medical Decision Analysis”, Management Science, Vol. 46 No. 11, pp. 1485-1496.

Camerer, C. and Ho, T.H. (1994), "Violations of Betweenness Axiom and Nonlinearity in Probability", Journal of Risk and Uncertainty, Vol.8 No. 2, pp. 167-196.

Devers, C., Wiseman, R. and Holmes, M. (2007), "The effects of endowment and loss aversion in managerial stock option valuation", Academy of Management Journal, Vol. 50 No. 1, pp. 191-208.

Dittmann, I. and Maug, E. (2007), "Lower salaries and no options? On the optimal structure of Executive pay", The Journal of Finance, Vol. 62 No. 1, pp. 303-343.

Dittmann, I., Maug, E. and Spalt, O.G. (2010), "Stick or Carrots? Optimal CEO Compensation when Managers are Loss Averse", Journal of Finance, Vol. 65, N. 6, pp. 2015-2050 
Hall, B. and Murphy, K. (2000), "Optimal Exercise prices for executive stock options”, American Economic Review, Vol. 90 No. 2, pp. 209-214.

Hall, B. and Murphy, K. (2002), "Stock options for undiversified executives", Journal of Accounting and Economics, Vol. 33 No. 2, pp. 3-42.

Hallock, K. and Olson, G. (2006), "The value of stock options to non-executive employees", Working Paper, No. 11950, National Bureau of Economic Research, Cambridge.

Heath, C., Huddart, S. and Lang, M. (1999), "Psychological factors and stock option exercise", Quarterly Journal of Economics, Vol. 114 No. 2, pp. 601-628.

Henderson, V. (2005), The impact of the market portfolio on the valuation, incentives and optimality of executive stock options, Quantitative Finance, Vol. 5 No. 1, pp. 35-47.

Hodge, F. Rajgopal, S. and Shevlin, T. (2006), "How do managers value stock options and restricted stock ?", Working Paper, University of Washington.

Holmstrom, B.R. and Milgrom, P.R. (1987), "Aggregation and linearity in provision of intertemporal incentives", Econometrica, Vol. 55 No. 2, pp. 303-328.

Huddart, S. and Lang, M. (1996), "Employee stock option exercises: an empirical analysis", Journal of Accounting and Economics, Vol. 21 No. 1, pp. 5-43.

Jensen, M. and Murphy, K.J. (1990), "Performance pay and top-management incentives", Journal of Political Economy, Vol. 98 No. 2, pp. 225-264.

Johnson, S.A. and Tian, Y.S. (2000), "The value and incentive effects of non-traditional executive stock option plans", Journal of Financial Economics, Vol. 57 No. 1, pp. 3-34.

Kahneman, D. and Tversky, A. (1979), "Prospect Theory: An analysis of decision under risk", Econometrica, Vol. 47 No. 2, pp. 263-292.

Lambert, R., Larcker, D. and Verrecchia, R. (1991), "Portfolio considerations in valuing Executive Compensation", Journal of Accounting Research, Vol. 29 No. 1, pp. 129-149.

Lambert, R. and Larcker, D. (2001), "How do employees value (often incorrectly) their stock options", available at: knowledge@wharton.

Sawers, K., Wright, A. and Zamora, V. (2006), "Loss aversion, stock-based compensation and managerial riskseeking behavior", paper presented at the AAA 2007 Management Accounting Section Meeting, available at: http://ssrn.com/ abstract=864224

Spalt, O.G. (2008), "Probability Weighting and Employee Stock Options", Working paper, Tilburg University, Department of Finance.

Thaler, R. (1999), "Mental Accounting Matters", Journal of Behavioral Decision Making, Vol. 12 No. 3, pp. 183206.

Tversky, A. and Kahneman, D. (1992), “Advances in Prospect Theory: Cumulative representation of uncertainty”, Journal of Risk and Uncertainty, Vol. 5 No. 4, pp. 297-323.

Wu, G. and Gonzalez, R. (1996), "Curvature of the Probability Weighting function”, Management Science, Vol. 42 No. 12, pp. 1676-1690. 